\title{
Maternal and foetal outcome of eclampsia in a referral hospital
}

\author{
Mousumi Das Ghosh*, Vinita Singh, Alokananda Ray
}

Department of Obstetrics and Gynecology, Tata Main Hospital, Jamshedpur, Jharkhand, India

Received: 12 January 2017

Accepted: 08 February 2017

\section{*Correspondence:}

Dr. Mousumi Das Ghosh,

E-mail: dr.mousumi@tatasteel.com

Copyright: $@$ the author(s), publisher and licensee Medip Academy. This is an open-access article distributed under the terms of the Creative Commons Attribution Non-Commercial License, which permits unrestricted non-commercial use, distribution, and reproduction in any medium, provided the original work is properly cited.

\section{ABSTRACT}

Background: Eclampsia is a life-threatening condition, common in developing countries with high fatality rate. It is a cause of maternal and foetal mortality and morbidity. The aim of the study is to determine the maternal and foetal outcome in Tata Main Hospital, Jamshedpur, Jharkhand, India.

Methods: All cases of eclampsia were analysed from January 2012 to December 2014 from admission to discharge or death of the patient. Age, parity, antenatal care, interval between attack and admission, blood pressure on admission, gestational age and mode of delivery were taken into account. There were 135 cases of eclampsia out of 14572 deliveries. Only singleton pregnancies were analysed.

Results: The incidence of antepartum eclampsia was $84.4 \%$, intrapartum $3 \%$ and postpartum $12.5 \%$. 83\% patients were primigravida, $33 \%$ less than $20 \mathrm{yrs}, 42 \%$ had no antenatal checkup and $45 \%$ had < 4 visits. Only $15 \%$ patients received magnesium sulphate before referral to the hospital. There were four maternal deaths and twenty-one perinatal deaths. Eleven patients needed ventilatory support, four developed pulmonary oedema, two patients had respiratory depression and three patients had renal failure.

Conclusions: Hypertensive disease in pregnancy requires proper antenatal care, early recognition and referral, adequate treatment and timely delivery.

Keywords: Eclampsia, Foetal, Maternal

\section{INTRODUCTION}

Eclampsia refers to the development of grand mal seizures in a woman with gestational hypertension or preeclampsia. ${ }^{1}$ This is a life-threatening condition, associated with significant risks- both maternal and foetal. The incidence of eclampsia in developed countries has been reported between $0.05 \%$ and $0.71 \%$, while in developing countries, rates are much higher. The complication rate in women with eclampsia has been reported to be as high as $35 \% .^{2}$ The maternal mortality rate (MMR) in India is 178 per 1,00,000 live births in December 2010. The rate of Bihar/Jharkhand is higher than the national average (219). As hypertensive disorders in pregnancy is a major contributing factor of maternal deaths, this study was carried out to determine the maternal and foetal outcome of eclampsia in our institution.

\section{METHODS}

This is a prospective study of maternal and foetal outcome of eclampsia carried out in a tertiary care centreTata Main Hospital, Jamshedpur, Jharkhand, India from January 2012 to December 2014. Method of recruitment was all consecutive cases of eclampsia treated in the hospital. The patients were admitted in labour ward cum Obstetric HDU which is adequately equipped, followed up during delivery, transfer to critical care unit, if any until discharge from hospital or death. Age of the patient, parity, antenatal care records, time interval between onset of seizures and admission, blood pressure on admission, 
gestational age of the patients and mode of delivery were taken into account. The gestational age was determined on the basis of history, antenatal records, clinical examination and, where available, ultrasound records. History was taken from family members, if patient was drowsy or unconscious during admission These patients were restrained with consent of family members. The baseline investigations done were complete blood count, platelet, liver function test, renal function test, electrolytes, coagulation profile. Urine albumin was done by dipstick method. The workup was to rule out other causes of seizures during pregnancy.

The type of eclampsia- antepartum, intrapartum and postpartum were studied along with maternal and foetal outcome. The birth weight was recorded for every baby delivered, even if stillborn. The gestational age at the time of seizure was recorded, and for postpartum seizures, this was taken to be the gestational age at delivery. Ultrasound was done in most cases, especially useful in suspected cases of IUFD. All women received Magnesium sulphate as anticonvulsant according to Pritchard regime. ${ }^{3}$ This was given for 24 hours postpartum or last convulsion (whichever is later), with clinical monitoring, that is periodic assessment of respiratory rate, knee jerk and urine output. The antihypertensive agent used was Labetalol in appropriate doses. Oral Nifedipine was the second drug of choice. Since many patients who were referred had a long convulsion to admission time, we followed a policy of early delivery after stabilization of the patient. If the patient was in active labour on admission, trial for vaginal delivery given. Caesarean section was done for all obstetric indications and where vaginal delivery was not imminent. Induction of labour had a role, in a stable patient with IUFD. However, induction delivery time was not allowed for more than 12 hours.

The maternal events taken into account were maternal death, respiratory depression, need for ventilator support, pulmonary oedema, pneumonia, renal failure, coagulopathy and CVA. Respiratory depression was diagnosed by clinical findings: respiratory rate $<12 /$ min and oxygen saturation $<92 \%$. Pulmonary oedema was diagnosed by clinical findings, pulse oximetry and chest $\mathrm{X}$ - ray. Pneumonia was diagnosed by clinical findings, chest X-ray and leucocytosis. Renal failure was diagnosed by oliguria and raised serum creatinine levels. Coagulopathy was diagnosed from bleeding manifestations and laboratory coagulation profile. ${ }^{4}$ For neonates, the events monitored were perinatal deaths (stillbirth and neonatal death within 7 days of birth), Apgar score $<7$ at 5 min and nursery admission $>7$ days. Length of hospital stay was also taken into account.

In atypical presentations such as prolonged post-ictal state, status epilepticus, gestational age $<20$ weeks or $>48$ hours postpartum or severe uncontrolled hypertension, CT scan/ MRI brain advised for.

\section{RESULTS}

Total singleton deliveries from Jan 2012 to Dec 2014 in Tata Main Hospital were 14,572. Out of which, 114 were cases of antepartum eclampsia, 4 cases of intrapartum eclampsia and 17 cases of postpartum eclampsia. The incidence was calculated to be $0.9 \%$. Primigravida contributed to $83 \%$ cases. $33 \%$ cases were less than 20 years and $45 \%$ patients were between 20-25 years. Most of these patients $(87 \%)$ either had no antenatal care or had <4 visits. Only 20 cases received magnesium sulphate before referral to our centre where as 85 cases were referred without the drug. Maximum patients had convulsion at term, only $18 \%$ were $<34$ weeks gestation. Convulsion to admission time was $>5$ hours in $59 \%$ cases, 1 to $5 \mathrm{hrs}$ in $30 \%$ and $<1$ hour in only $11 \%$ cases. As most of the patients took $>5$ hrs after convulsion to reach hospital, delivery was expedited after stabilization of the patient. LSCS was done in $73 \%$ patients and vaginal delivery in the rest (Table 1).

Table 1: Comparison of baseline characteristics.

\begin{tabular}{|c|c|c|}
\hline Parameter & Total cases $(n=135)$ & $\%$ \\
\hline \multicolumn{3}{|l|}{ Age } \\
\hline$<20$ & 45 & 33 \\
\hline $20-25$ & 61 & 45 \\
\hline $25-30$ & 22 & 16 \\
\hline$>30$ & 7 & 6 \\
\hline \multicolumn{3}{|l|}{ Parity } \\
\hline Primi & 112 & 83 \\
\hline Multi & 23 & 17 \\
\hline \multicolumn{3}{|c|}{ Antenatal care received } \\
\hline Regular ANC & 18 & 13 \\
\hline$<4$ visits & 61 & 45 \\
\hline No visits & 56 & 42 \\
\hline \multicolumn{3}{|c|}{ Convulsion to admission time } \\
\hline$<1$ hour & 15 & 11 \\
\hline $1-5$ hours & 40 & 30 \\
\hline$>5$ hours & 80 & 59 \\
\hline \multicolumn{3}{|c|}{ Gestational age } \\
\hline$<34$ weeks & 24 & 18 \\
\hline 34-37 weeks & 40 & 30 \\
\hline 37-40 weeks & 55 & 41 \\
\hline$>40$ weeks & 16 & 11 \\
\hline \multicolumn{3}{|c|}{ Mode of delivery } \\
\hline Vaginal & 21 & 16 \\
\hline instrumental & 15 & 11 \\
\hline LSCS & 99 & 73 \\
\hline \multicolumn{3}{|l|}{ Birth weight } \\
\hline$<1.5 \mathrm{~kg}$ & 13 & 10 \\
\hline 1.5 to $2.5 \mathrm{~kg}$ & 62 & 45 \\
\hline 2.5 to $4 \mathrm{~kg}$ & 60 & 45 \\
\hline$>4 \mathrm{~kg}$ & 0 & 0 \\
\hline
\end{tabular}

In present study, there were four maternal deaths, four had pulmonary oedema and two patients each of pneumonia and respiratory depression. Eleven needed ventilatory support, two had cerebro-vascular accidents 
managed by neurophysicians. Two patients developed status eclampticus, managed with phenytoin and diazepam in critical care unit (Table 2).

Table 2: Maternal and foetal outcome.

\begin{tabular}{|lllll|}
\hline Maternal outcome & Antepartum & Intrapartum & Postpartum & Total \\
\hline Maternal death & 4 & 0 & 0 & 4 \\
\hline Pulmonary oedema & 4 & 0 & 0 & 4 \\
\hline Pneumonia & 1 & 0 & 0 & 2 \\
\hline Respiratory depression & 2 & 0 & 1 & 2 \\
\hline Ventilatory support & 10 & 0 & 0 & 11 \\
\hline Renal failure & 3 & 0 & 0 & 3 \\
\hline Coagulopathy & 2 & 0 & 0 & 2 \\
\hline CVA & 1 & 0 & 0 & 1 \\
\hline Status eclampticus & 2 & 0 & 0 & 2 \\
\hline Sepsis & 2 & 0 & & 7 \\
\hline Blood transfusion & 7 & 0 & 0 & 2 \\
\hline Maternal outcome & & & 0 & 21 \\
\hline Perinatal death & 21 & 0 & 2 & 16 \\
\hline Apgar score $<7$ at 5 min & 16 & 0 & 0 & 27 \\
\hline Nursery admission $>$ days & 25 & 0 & & \\
\hline
\end{tabular}

For overlapping complications, taken into account only once.

There were seventeen cases of intrauterine foetal death (on admission) and four cases of early neonatal deaths due to meconium aspiration (two cases) and prematurity (two cases). There were sixteen babies with 5 minutes APGAR less than 7. All were cases of antepartum eclampsia. Thirty-five babies of antepartum eclampsia needed nursery admission more than a week and two babies of postpartum eclampsia needed so. Two babies had late neonatal deaths due to sepsis while the rest were discharged after recovery.

CT scan/MRI was advised for 16 patients with atypical eclampsia. Reports were multi infarct in 2 cases, posterior reversible encephalopathy syndrome (PRES) in 2 cases, normal report in 8 cases. Four patients refused for monetary reasons. All of them recovered without neurological deficit.

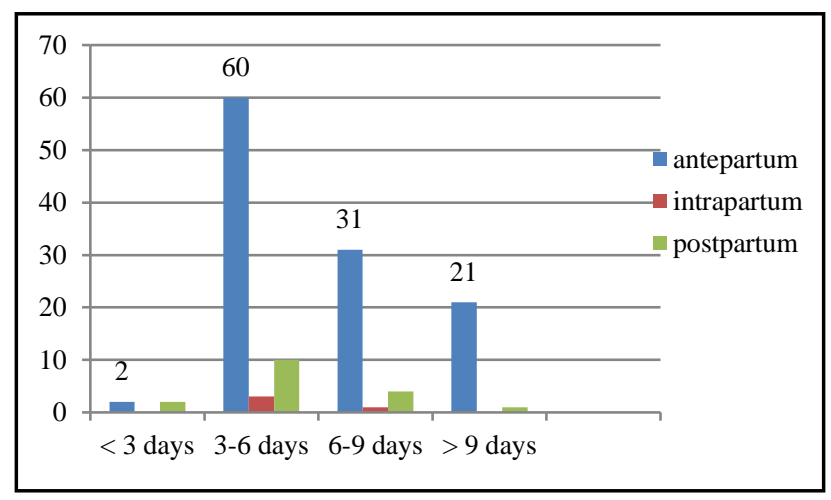

Figure 1: Length of stay.

\section{DISCUSSION}

The incidence of antepartum eclampsia in our study was $84.4 \%$, intrapartum $3 \%$ and postpartum $12.5 \%$. The frequency rate was 1 in 108. Only singleton pregnancies were analysed. According to millennium development goals target 5.B, universal access to reproductive health is promoted. The World Health Organization has recommended a minimum of four antenatal care visits to ensure the well-being of mothers and new born. ${ }^{5}$ A large group of patients- $42 \%$ had no antenatal care and $45 \%$ had $<4$ visits. These patients had the highest complications and bad maternal and foetal outcome.

$48 \%$ of deliveries were preterm. $83 \%$ of patients were primigravida. $33 \%$ patients were less than 20 years. Mean age was 21 years. Teenage pregnancy was found to be a risk factor. In a study by Adamu in Nigeria, $76 \%$ patients were primigravida with no antenatal care, mean age was 21 years. ${ }^{6}$ Caesarean section rate in present study was $73 \%$. This was because of prolonged convulsion to admission time and our policy of early delivery for effective maternal resuscitation. This is comparable to a study by Akhtar R in Bangladesh where $60 \%$ patients had no antenatal check-up, 64\% had antepartum eclampsia, $63 \%$ were delivered by LSCS. ${ }^{7}$

The high incidence of eclampsia was due to referral of cases from peripheral hospitals. This is a referral hospital and treats cases of East Singhbhum, West Singhbhum, Sareikela- kharsawan (Jharkhand), Mayurbhanj, Sundergarh (Orissa) and Purulia, Medinipur (West Bengal). The hospital has 18 bedded labour ward cum 
obstetric high dependency unit (HDU) manned by specialists around the clock and a team of experienced sisters. Critical care unit with ventilatory support is available in the hospital. The neonatal care unit is also well equipped with ventilatory support to take care of the sick newborns. Only 20 cases received magnesium sulphate before referral to our center where as 85 cases were referred without the drug. The patients who received magnesium sulphate before referral had the best maternal and foetal outcomes. ${ }^{8}$ This reflects poor awareness amongst healthcare providers. There were four maternal deaths. All of them were from remote villages and had no antenatal check-up in pregnancy. They were cases of antepartum eclampsia with Intrauterine foetal death, and preterm, referred from health centers without magnesium sulphate. LSCS was done for early delivery and effective maternal resuscitation. They needed ventilatory support. One patient expired within 5 hours of admission due to pulmonary edema. The other three were on ventilator for a prolonged period and had multi organ failure. All women who died in our study had multiple convulsions and were in a comatose state at the time of admission. This reflects that cases of antepartum eclampsia were more severe than those occurring at or after delivery.

Excluding maternal deaths, the other maternal morbidities were- pulmonary edema in four cases of antepartum eclampsia, two cases of pneumonia (one antepartum and other postpartum), two cases of respiratory depression (antepartum) and eleven patients needed ventilatory support (10 antepartum and one postpartum). Renal failure in three cases needed dialysis (antepartum), coagulopathy in two cases, status eclampticus in two cases and one patient of antepartum eclampsia had cerebrovascular accident. These cases were managed by a multidisciplinary team of anaesthetists, intensivists, neurophysicians and haematologist. All these patients recovered and were discharged in good health.

There were twenty-one perinatal deaths (Fifteen cases of intrauterine foetal death and six cases of early neonatal deaths). All perinatal deaths were cases of antepartum eclampsia. Sixteen babies with APGAR score $<7$ at 5 min were cases of antepartum eclampsia. Twenty-five babies of antepartum eclampsia and two babies of postpartum eclampsia needed nursery admission for more than a week. Reasons were birth asphyxia, prematurity and sepsis. All these figures reflect poor foetal outcome in cases of antepartum eclampsia. In a study conducted in Nigeria, fifty percent of perinatal deaths were stillbirths and all these from unbooked mothers. ${ }^{9}$

Length of hospital stay was taken into account. 3\% patients were discharged within three days of admission, $54 \%$ within 3 to 6 days, $27 \%$ within 6 to 9 days and $16 \%$ had length of stay more than 9 days (Figure 1). This reflects the financial burden of the disease.

\section{CONCLUSION}

Eclampsia is associated with poor maternal and foetal outcome. They are more likely to be delivered by Caesarean section. Antepartum eclampsia has higher rate of complications compared to others.

Recommendations to reduce the burden of eclampsia are promoting and improving quality of health education especially in third trimester, increasing access to high quality essential obstetric care and improving the service delivery in rural areas. Hypertensive disease in pregnancy requires proper antenatal care, early recognition and referral, adequate treatment and timely delivery. magnesium sulphate should be made available in all health centres, so that loading dose can be given before referral to a higher centre. Clear protocols for management of hypertension in pregnancy at all levels of health care are required for better maternal and perinatal outcome. ${ }^{10}$

\section{Funding: No funding sources Conflict of interest: None declared \\ Ethical approval: The study was approved by the Institutional Ethics Committee}

\section{REFERENCES}

1. Ghulmiyyah L, Sibai B. Maternal Mortality from preeclampsia/eclampsia. Semin Perinatol. 2012;36(1):56-9.

2. Lal AK, Gao W, Hibbard JU. Eclampsia: maternal and neonatal outcomes: Int $\mathrm{J}$ Women Cardiovascular Health. 2013; 3;186-90.

3. Roy J, Mitra JK, Pal A. Magnesium sulphate versus Phenytoin in eclampsia- maternal and foetal outcome- a comparative study. AMJ. 2013;6(9)483-95.

4. Seal SL, Ghosh D, Kamilya G. Does route of delivery affect maternal and perinatal outcome in women with eclampsia? A randomized controlled pilot study. Am J Obstet Gynecol. 2012;206:484.e1-7.

5. The millennium development goals report 2014, United Nations; New York: 2014.

6. Adamu AN. Pregnancy outcome in women with eclampsia at a tertiary centre in northern Nigeria: Afr J Med Med Sci. 2012:41(2):211-9.

7. Akhtar R, Ferdous A, Bhuiyan SN. Maternal and fetal outcome of eclamptic patients in a tertiary hospital. Bangladesh J Obstet Gynaecol. 2011;26(2):77-80.

8. Joshi SD, Veerendrakumar CM. Single dose MgSo4 Regimen for Eclampsia- A Safe Motherhood Initiative. J Clin Diagnost Res. 2013;7(5):868-72.

9. George IO, Jeremiah I. Perinatal outcome of babies delivered to eclamptic mothers: a prospective study from a Nigerian tertiary hospital. Int J Biomed Sci. 2009;5(4):390-4.

10. Bailit JL, Grobman WA, McGee P, Reddy UM, Wapner $\mathrm{RJ}$, Varner MW, et al. Is the use of protocols for obstetric care associated with better outcomes? Am J Obstet Gynaecol. 2015;213(1):86.

Cite this article as: Das Ghosh M, Singh V, Ray A. Maternal and foetal outcome of eclampsia in a referral hospital. Int J Reprod Contracept Obstet Gynecol 2017;6:1021-4. 